MIDPI

\title{
sciforum
}

\section{Evaluation of Different Scoring Functions for Docking and Virtual Screening against GPCR Drug Targets}

\author{
Tatiana F. Vieira, Rita P. Magalhães, Nuno M. F. S. A. Cerqueira, Sérgio F. Sousa
}

UCIBIO/REQUIMTE, BioSIM Departamento de Biomedicina, Faculdade de Medicina, Universidade do Porto, Alameda Professor Hernâni Monteiro,

4200-319 Porto, Portugal

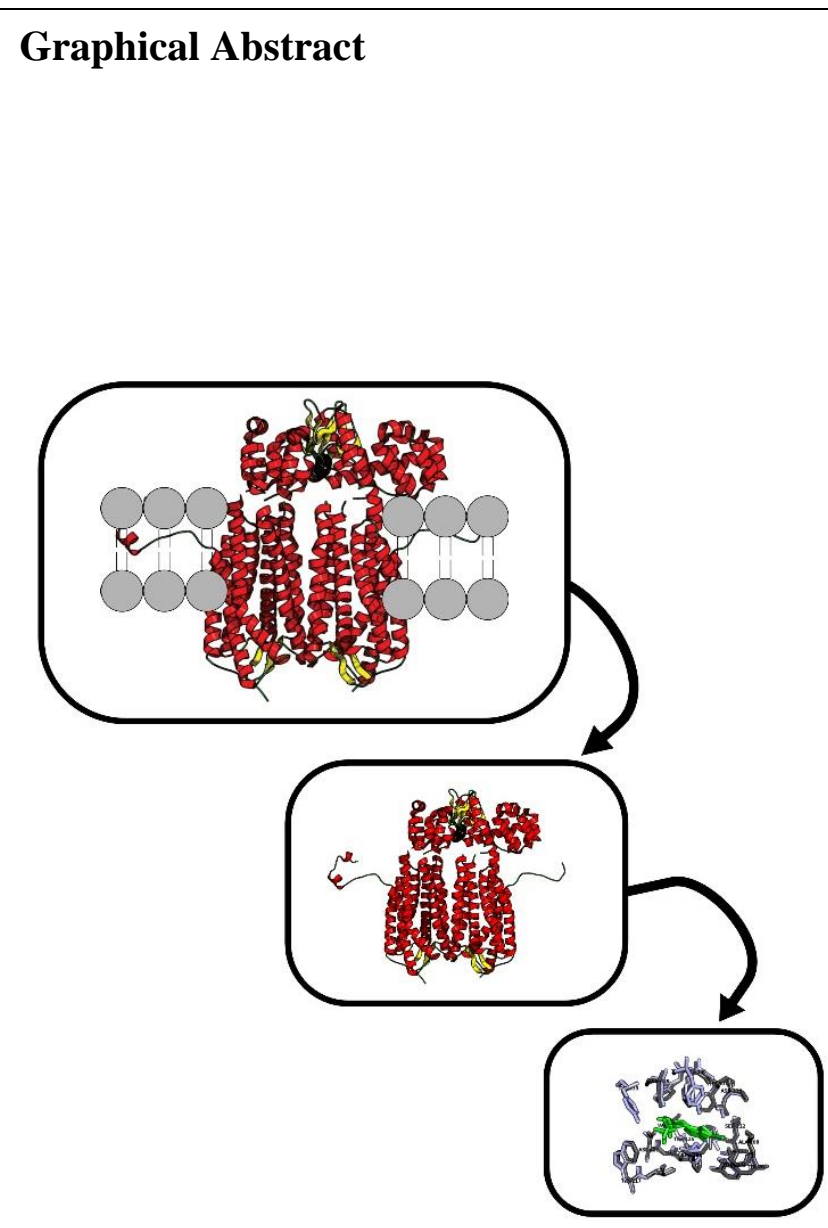

Abstract.

G-protein-coupled receptors (GPCRs) constitute a large family of structurally similar proteins that respond to diverse physiological and environmental stimulants and that includes many therapeutic targets. In fact, $40 \%$ of all modern medicinal drugs are thought to target G-proteincoupled receptors (GPCRs), making this large family of proteins a particular appealing target for drug discovery efforts [1, 2].

Protein-ligand docking is a computational method that tries to predict and rank the structure resulting from the association between a ligand and a target protein [3]. Virtual screening (VS) can use docking to evaluate databases with millions of compounds to identify promising new molecules that could bind to a specific target of pharmacological interest, including GPCRs [4]. This strategy if often used to limit the amount of molecules that has to be tested experimentally and to reduce the cost in the identification of new lead molecules for drug development.

This work reports a detailed comparison of the popular Autodock [5] and Vina [6] software programs in ligand/decoys discrimination against 5 GPCR proteins, (Adenosine $2 a$ receptor, Beta-1 adrenergic receptor, Beta-2 adrenergic receptor, $C-X-C$ chemokine receptor 
type 4 and Dopamine $D 3$ receptor), for a total of 1480 ligands and 99763 decoys. The results show that AutoDock is more efficient in recovering real ligands among the top $1 \%$ solution than VINA, when applying virtual screening to GPCR receptors.

\section{Conclusions}

The results show that AutoDock is more efficient in recovering real ligands among the top 1\% solution than VINA, when applying virtual screening to GPCR receptors. However, the results illustrate that AutoDock and Vina have different strengths and weaknesses, with a performance that can vary significantly with the type of protein target, and with the specific characteristics of the ligands (size, flexibility, etc).

These results also highlight the need to evaluate, a priori, the accuracy of the docking software for the specific protein target, or family of targets, before embarking on a virtual screening study..

\section{References}

1. Lagerstrom, M.C. and H.B. Schioth, Nature Reviews Drug Discovery, 2008. 7(4), 339-357.

2. Overington, J.P., B. Al-Lazikani, and A.L. Hopkins, Opinion - How many drug targets are there? Nature Reviews Drug Discovery, 2006. 5(12): p. 993-996.

3. Sousa, S.F., P.A. Fernandes, and M.J. Ramos, Protein-ligand docking: Current status and future challenges. Proteins-Structure Function and Bioinformatics, 2006. 65(1): p. 15-26.

4. Shoichet, B.K. and B.K. Kobilka, Structure-based drug screening for G-protein-coupled receptors. Trends in Pharmacological Sciences, 2012. 33(5): p. 268-272.

5. Morris, G. M., Huey, R., Lindstrom, W., Sanner, M. F., Belew, R. K., Goodsell, D. S. and Olson, A. J. (2009) Autodock4 and AutoDockTools4: automated docking with selective receptor flexiblity. J. Computational Chemistry 2009, 16: 2785-91.

6. Trott, O., Olson, A.J., AutoDock Vina: improving the speed and accuracy of docking with a new scoring function, efficient optimization and multithreading, Journal of Computational Chemistry 31 (2010) 455-461 